biopsies for pleural tuberculosis. Thorax 2010;65: 857-862.

2. Diacon AH, Van de Wal BW, Wyser C, et al. Diagnostic tools in tuberculous pleurisy: a direct comparative study. Eur Respir J 2003;22:589-91.
3. Haybittle J. Repeated assessment of results in clinical trials of cancer treatment. Br J Radiol 1971:44:793-7.

4. Peto R, Pike M, Armitage P. Design and analysis of randomized clinical trials requiring prolonged observation of each patient: introduction. $\mathrm{Br} J$ Cancer 1976:34:585-612.

5. Maskell NA, Davies CW, Nunn AJ, et al. U.K. Controlled trial of intrapleural streptokinase for pleural infection. N Engl J Med 2005;352:865-74.

\title{
Severity scores for CAP. 'Much workload for the next bias'
}

\section{Santiago Ewig, ${ }^{1}$ Antoni Torres ${ }^{2}$}

Tools for the assessment of severity of patients with community-acquired pneumonia (CAP) have attracted much interest in the recent past. In this issue of Thorax (see page 878) two systematic reviews and meta-analyses address the value of such tools including no less than $40^{1}$ and 23 studies. $^{2}$ Despite different inclusion strategies and statistical approaches, both studies report two main and similar conclusions. First, both the most extensively investigated tools (Pneumonia Severity Index (PSI) and CURB-65/CRB65) have remarkably favourable power to predict mortality. Secondly, whereas PSI is somewhat better in predicting patients at low risk, the reverse is true for CURB-65/ CRB-65. However, these differences are of questionable clinical relevance. Thus, both tools can be regarded as equivalent. CRB65 is the simplest tool and can easily be remembered and also applied in the outpatient setting. These straightforward conclusions are the result of a decade of intensive and successful work to establish clinically useful tools of severity assessment. So far success is impressive; clinicians now can use a very simple tool as an adjunct to clinical judgement, and studies on patients with CAP can rely on a validated tool for severity stratification. ${ }^{3}$

At this point, it is worthwhile having a look at the perspectives beyond: where do we have to go from here? Actually, there are many issues that still must be

\footnotetext{
${ }^{1} T$ Thoraxzentrum Ruhrgebiet, Kliniken für Pneumologie und Infektiologie, EVK Herne und

Augusta-Kranken-Anstalt Bochum, Germany; ${ }^{2}$ Servei de Pneumologia, Institut Clinic del Tórax, Hospital Clinic de Barcelona, Facultad de Medicina, Universitat de Barcelona, Idibaps, Ciber de Enfermedades Respiratorias (CIBERES), Spain

Correspondence to Prof. Dr. Santiago Ewig, Thoraxzentrum Ruhrgebiet, Kliniken für Pneumologie und Infektiologie, EVK Herne und Augusta-Kranken-Anstalt Bochum, Bergstrasse 26, D-44791 Bochum, Germany; ewig@augusta-bochum.de
}

addressed concerning validation in intervention studies and still insufficently recognised ambiguities inherent to the severity scores.

PSI has been prospectively validated in independent populations as a tool to guide site of treatment decisions, and the use of the PSI was associated with a larger proportion of patients in PSI risk classes I and II who were treated in the outpatient environment without compromising their safety. ${ }^{4}$ No such studies have been performed for CURB-65/CRB-65, and obviously these studies need to be performed.

On the other hand, it is difficult to imagine that such a complex tool as the PSI will ever be implemented in routine clinical practice, particularly in the outpatient setting. CURB-65, and particularly CRB-65, is clearly superior in terms of simplicity, and it is its simplicity that makes it preferable even if it provides slightly inferior predictions. However, the four-variable CRB-65 score is a delicate tool and vulnerable to ambiguities of its components. First, the respiratory rate may be difficult to assess in patients with rapid shallow breathing. In fact, no rules have been established regarding how to assess the respiratory rate precisely. Secondly, pneumonia-associated confusion is difficult if not impossible to distinguish from premorbid mental deficiencies in elderly and disabled patients. Evidently, pre-existing and pneumoniarelated confusion cannot be confidently differentiated in every case. As a result, confusion cannot be regarded exclusively as a parameter reflecting only severe sepsis but must also be recognised as a parameter possibly reflecting pre-existing mild central nervous system co-morbidity. The substrate of the parameter 'confusion' remains somewhat vague. Thirdly, if CURB-65 is used, blood urea nitrogen may be a confounder in patients with preexisting renal insufficiency and in the elderly. In fact, some have found that CURB-65 works less well in elderly patients. In one recent study of hospitalised elderly patients, the area under the curve (AUC) was significantly higher for the $<65$-year cohort in comparison with older patients (0.93 vs 0.7$).{ }^{5}$ Others have suggested that oxygenation instead of confusion and blood urea nitrogen might work at least as well. ${ }^{6}$ There is evidence that performance status is an independent predictor for short- and long-term mortality in hospitalised elderly patients. ${ }^{7}$ Accordingly, combining the modified PSI with performance status led to better predictions being obtained. ${ }^{8}$ Fourthly, the threshold of age (65 years) seems arbitrary and there is some evidence that higher cut-offs might work better. ${ }^{9}$ Splitting age into decades might be even more promising. However, the rule would clearly lose much of the main strength of simplicity if stratification by age were to form a part of it.

The simplicity of the CRB-65 rule is not without pitfalls. A score of CRB-65 = 1 already implies an increased risk of death and should prompt consideration of hospitalisation. However, when this score is applied in primary care, hospital referral would have to be considered in all patients just because of an an age older than 65 years. Although increasingly age $>50$ years is associated with increased risk of death, it is clearly impractical and inadequate to hospitalise all patients with CAP only because they are older than 65 years. ${ }^{10}$ Thus, it is important to exclude age alone as a criterion when CRB-65 is used as an aid for the decision to hospitalise.

Studies addressing severity assessment of CAP mainly refer to hospitalised patients, and clearly more data are needed for the outpatient setting. Obviously, the available tools do not measure the same things and are imperfect, and there has been insufficient attention so far paid to the reasons behind this. Several studies have incorporated biomarkers to improve mortality predictions, and as things stand today it appears that some of these (in particular C-reactive protein and ${ }^{11} 12$ procalcitonin, ${ }^{11}$ but also adrenomedullin) ${ }^{13-15}$ should be able to meet expectations. 
Limited data are available for specific important subgroups of patients with pneumonia such as patients with cancer, those receiving haemodialysis and those residing in nursing homes. Settings different from that of the derivation study may result in different predictions of the severity tools. Finally, as stated by Chalmers et al, impact analyses are lacking to investigate the impact of severity tools on predefined outcomes. ${ }^{1}$

These perspectives are challenging, and deserve much attention in the future. Nevertheless, rethinking the potentials and limitations of severity scores for pneumonia, there are two areas of uncertainty which need to be addressed. The first relates to the adequate use of severity scores as an aid for the decision to hospitalise. In younger and/or not severely disabled patients, things are quite straightforward: the more severe condition with which a patient with pneumonia presents, the clearer is the indication to hospitalise. This is quite different from the far more complex situation of elderly and disabled patients. It is well described that the functional status of patients with CAP may worsen after having been hospitalised. ${ }^{7} 1617$ This worsening is associated with severity of pneumonia and premorbid performance status. ${ }^{7}$ Some of this decline may be ascribed to hospitalisation per se and may be preventable. In fact, 'hospital at home care' has been shown to be feasible and to be associated with fewer complications. ${ }^{18}$ In another study, patients treated with 'hospital at home care' experienced modest improvements in performance scores, whereas those treated in a traditional acute care hospital declined. Likewise, a greater proportion of 'hospital at home care' patients improved in function and smaller proportions declined or had no change in functional status. ${ }^{19}$ Finally, a considerable proportion of severely disabled patients may be candidates for restriction on treatment escalations and are better treated where they reside, provided adequate palliative care can be offered. All these considerations should lead to the investigatation of which elderly patients with CAP truly benefit from hospitalisation and to include the assessment of function before and during hospitalisation as an integral part of clinical evaluation of these patients. Moreover, models of 'hospital at home care' carry an enormous potential for improvements in the care of elderly and disabled patients and should be further evaluated in terms of efficacy and cost-effectiveness. ${ }^{18}$
The second important issue relates to the assessment of severe CAP. Neither PSI nor CURB-65/CRB-65 is an adequate predictor of the need for intensive care treatment, either in the intensive care unit or in intermediate care settings. ${ }^{19-21}$ Therefore, additional parameters and scores have been derivated and validated, and it appears that the modified American Thoracic Society (ATS) score as well as the most recent Infectious Diseases Society of America (IDSA)/ATS criteria are better predictors of the need for intensive care treatment. ${ }^{22-25}$ Nevertheless, they both remain imperfect, and additional severity scores seem impractical (Ewig $\mathrm{S}$ et al unpublished data 2010). Therefore, we need to look for parameters which can be incorporated into the CURB-65/CRB-65 scores and which improve the predictions of the need for intensive care treatment without compromising overall risk predictions (particularly low risk predictions) and the simplicity of these scores. Oxygenation as assessed by oximetry is a compelling variable which may bring about substantial independent predictive power to such a severity score. Again, biomarkers may be of help at least in the emergency department and/or hospital setting. As the German poet Bertolt Brecht said: 'I have much workload, I am preparing my next bias'.

Competing interests None.

Provenance and peer review Commissioned; externally peer reviewed.

Thorax 2010;65:853-855

doi:10.1136/thx.2010.143297

\section{REFERENCES}

1. Chalmers JD, Singanayagam A, Akram AR, et al. Severity assessment tools for predicting mortality in hospitalised patients with community-acquired pneumonia. Systematic review and meta-analysis. Thorax 2010;65:878-83.

2. Loke YK, Kwok CS, Niruban A, et al. Value of severity scales in predicting mortality from community-acquired pneumonia: systematic review and meta-analysis. Thorax 2010;65:866-72.

3. Ewig S, Welte T. CRB-65 for the assessment of pneumonia severity: who could ask for more? Thorax 2008;63:665-6.

4. Renaud B, Coma E, Labarere J, et al; Pneumocom Study Investigators. Routine use of the Pneumonia Severity Index for guiding the site-of-treatment decision of patients with pneumonia in the emergency department: a multicenter, prospective, observational, controlled cohort study. Clin Infect Dis 2007:44:41-9.

5. Parsonage M, Nathwani D, Davey $P$, et al. Evaluation of the performance of CURB-65 with increasing age. Clin Microbiol Infect 2009;15:858-64.

6. Myint PK, Kamath AV, Vowler SL, et al; British Thoracic Society. Severity assessment criteria recommended by the British Thoracic Society (BTS) for community-acquired pneumonia (CAP) and older patients. Should SOAR (systolic blood pressure, oxygenation, age and respiratory rate) criteria be used in older people? A compilation study of two prospective cohorts. Age Ageing 2006;35:286-91.

7. Torres $\mathbf{O H}$, Muñoz J, Ruiz D, et al. Outcome predictors of pneumonia in elderly patients: importance of functional assessment. J Am Geriatr Soc 2004;52:1603-9

8. Naito T, Suda T, Yasuda K, et al. A validation and potential modification of the pneumonia severity index in elderly patients with communityacquired pneumonia. J Am Geriatr Soc 2006;54:1212-19

9. Teramoto $\mathbf{S}$, Yamamoto $\mathrm{H}$, Yamaguchi $\mathrm{Y}$, et al. Lower respiratory tract infection outcomes are predicted better by an age $>80$ years than by CURB-65. Eur Respir J 2008;31:477-8.

10. Bont J, Hak E, Hoes AW, et al. Predicting death in elderly patients with community-acquired pneumonia: a prospective validation study reevaluating the CRB-65 severity assessment tool. Arch Intern Med 2008;168:1465-8.

11. Krüger S, Ewig S, Marre R, et al; CAPNETZ Study Group. Procalcitonin predicts patients at low risk of death from community-acquired pneumonia across all CRB-65 classes. Eur Respir J 2008;31:349-55.

12. Menéndez R, Martínez R, Reyes S, et al. Biomarkers improve mortality prediction by prognostic scales in community-acquired pneumonia. Thorax 2009;64:587-91.

13. Christ-Crain M, Morgenthaler NG, Stolz D, et al. Pro-adrenomedullin to predict severity and outcome in community-acquired pneumonia. Crit Care 2006;10:R96.

14. Huang DT, Angus DC, Kellum JA, et al. Midregional proadrenomedullin as a prognostic tool incommunityacquired pneumonia. Chest 2009;136:823-31.

15. Krüger S, Ewig S, Giersdorf S, et al. Cardiovascular and Inflammatory Biomarkers to Predict Short- and Long-term Survival in Community-acquired Pneumonia. Am J Respir Crit Care Med. 2010 Jul 16. [Epub ahead of print] PubMed PMID: 20639437a. Am J Respir Crit Care Med. 2010 Jul 16. [Epub ahead of print] PubMed PMID: 20639437

16. Covinsky KE, Palmer RM, Fortinsky RH, et al. Loss of independence in activities of daily living in older adults hospitalized with medical illnesses: increased vulnerability with age. J Am Geriatr Soc 2003;51:451-8.

17. Mody L, Sun R, Bradley SF. Assessment of pneumonia in older adults: effect of functional status. J Am Geriatr Soc 2006;54:1062-7.

18. Leff B, Burton L, Mader SL, et al. Hospital at home: feasibility and outcomes of a program to provide hospital-level care at home for acutely ill older patients. Ann Intern Med 2005;143:798-808.

19. Leff B, Burton L, Mader SL, et al. Comparison of functional outcomes associated with hospital at home care and traditional acute hospital care. J Am Geriatr Soc 2009:57:273-8.

20. Ewig S, de Roux A, Bauer T, et al. Validation of predictive rules and indices of severity for community acquired pneumonia. Thorax 2004;59:421-7.

21. Angus DC, Marrie TJ, Obrosky DS, et al. Severe community-acquired pneumonia: use of intensive care services and evaluation of American and British Thoracic Society diagnostic criteria. Am J Respir Crit Care Med 2002;166:717-23.

22. Buising $\mathbf{K L}$, Thursky KA, Black JF, et al. A prospective comparison of severity scores for identifying patients with severe community acquired pneumonia: reconsidering what is meant by severe pneumonia. Thorax 2006:61:419-24.

23. Niederman MS, Mandell LA, Anzueto A, et al Guidelines for the management of adults with community-acquired pneumonia. Diagnosis, assessment of severity, antimicrobial therapy, and 
prevention. Am J Respir Crit Care Med 2001:163:1730-54.

24. Mandell LA, Wunderink RG, Anzueto A, et al. Infectious Diseases Society of America/American

Thoracic Society consensus guidelines on the management of community-acquired pneumonia in adults. Clin Infect Dis 2007:44(Suppl 2): S27-72.

25. Liapikou A, Ferrer M, Polverino E, et al. Severe community-acquired pneumonia: validation of the Infectious Diseases Society of America/ American Thoracic Society guidelines to predict an intensive care unit admission. Clin Infect Dis 2009;48:377-85.

\title{
Hot off the breath: the 2009 H1N1 flu pandemic may be gone but should not be forgotten
}

\author{
David R Thickett, Mark Griffiths, Gavin D Perkins, \\ Danny F McAuley, on behalf of the UK and Ireland \\ Acute Lung Injury Group
}

The 2009 influenza pandemic was caused by the emergence of a new influenza virus, pandemic H1N1 2009 influenza A (pH1N1), to which many people had no pre-existing immunity. It caused unusual and extensive outbreaks of disease in the summer months in many countries and very high levels of disease in the winter months. The pandemic virus had almost complete dominance over other seasonal influenza viruses and was unusual in its clinical presentation because the most severe cases occurred more often in younger age groups. The WHO and the world's healthcare systems mobilised a massive healthcare resource effort to prepare for the potentially devastating effects of an influenza pandemic. In the UK there have been 474 deaths reported associated with confirmed cases of pH1N1 since the beginning of the pandemic. While this is almost certainly a sizeable underestimate, fortunately it is a figure much lower than some initial predictions. $^{1}$

As of 10 August 2010, the WHO announced that the pandemic was over. In the southern hemisphere $\mathrm{pH} 1 \mathrm{~N} 1$ rates remain well below those observed during the same period in 2009 during the first pandemic wave. No large and unusual summer outbreaks have occurred in either northern or southern hemispheres. Indeed, the seasonal influenza A (H3N2) and

Lung Investigation Unit, Queen Elizabeth Hospital, Birmingham, UK

Correspondence to Dr David Thickett, Lung Investigation Unit, $1^{\text {st }}$ Floor, Nuffield House, Queen Elizabeth Hospital, Birmingham B15 2TH, UK; d.thickett@bham.ac.uk influenza B viruses are currently being reported in many countries. Based on this overall picture, the WHO believes that the evidence is strong that the recent influenza pandemic patterns are transitioning towards seasonal patterns of influenza. ${ }^{2}$

What has been learnt about pH1N1induced respiratory failure from the 2009 experience? While pH1N1 disease is usually mild and often subclinical, it may cause life-threatening respiratory failure that evolves rapidly causing classical acute respiratory distress syndrome with a high incidence of other organ failures. Over the course of 2009, UK hospital admissions associated with influenza were increased sevenfold and, at the peak of the pandemic, almost 200 intensive care beds were occupied with influenza cases. Fortunately, perhaps because the patients were young, the ICU outcomes have been good with approximately $80 \%$ survival in multiple series despite the requirement for advanced support techniques including extracorporeal membrane oxygenation. ${ }^{3}$

Although roughly two-thirds of patients admitted to ICUs across the world had no longstanding health problems, several groups have been reported to be at increased risk of severe disease. The commonest comorbidities were asthma or chronic obstructive pulmonary disease, diabetes mellitus, heart disease and sickle cell disease. ${ }^{4}$ A surprising proportion of critically ill patients were also obese or pregnant. While morbid obesity (body mass index $>40 \mathrm{~kg} / \mathrm{m}^{2}$ ) increased the risk of hospitalisation more than fourfold, an effect on mortality has not been demonstrated so far. ${ }^{5}$ In contrast, infection during pregnancy or the postpartum period was associated with a poor outcome from $\mathrm{pH} 1 \mathrm{~N} 1$, as has been shown for other forms of influenza. ${ }^{6}$

Predictions from experience with previous pandemics indicate that $\mathrm{pH} 1 \mathrm{~N} 1$ will continue to circulate for several years. Major concern remains in relation to the potential for the virus to mutate to a more lethal form. This concern is based on data suggesting previous influenza pandemics emerged after viral evolution ${ }^{7}$ and with recent reports confirming that the $\mathrm{pH} 1 \mathrm{~N} 1$ virus has undergone genetic reassortment. ${ }^{8}$ Indeed, there have been suggestions that mutant strains (notably D222G isolated from fatal cases in Norway) have a predilection for causing more aggressive disease, possibly because they bind preferentially to the glycan cell surface receptor that is highly expressed in the lower respiratory tract. $^{9}$

While the rates of $\mathrm{pH} 1 \mathrm{~N} 1$ infection are now much lower, localised outbreaks may show significant levels of $\mathrm{pH} 1 \mathrm{~N} 1$ transmission. The risk of severe illness caused by $\mathrm{pH} 1 \mathrm{~N} 1$ remains, and with it a need for hospitalisation and critical care support. Indeed, within $48 \mathrm{~h}$ of the WHO downgrading the pandemic status, five patients with suspected pH1N1 were admitted to a hospital in the West Midlands. Two of these patients rapidly deteriorated with bilateral pulmonary infiltrates on chest $\mathrm{x}$ ray requiring admission to the ICU and initiation of mechanical ventilation. The first case was a 44-year-old obese woman with a history of alcohol abuse and asthma who needed ventilation for 4 days. The second case was a 52-year-old man with treated vasculitis and a history of asthma and diabetes. Both patients met the criteria for acute lung injury upon intubation, with the second case progressing to ARDS on day 2 after intubation. Clearly, both these cases had more than one comorbid condition associated with severe $\mathrm{pH} 1 \mathrm{~N} 1$ infection. ${ }^{4}$ These cases highlight the need for maintained vigilance, a high index of suspicion and a low threshold for empirical treatment of highrisk groups as we enter the seasonal influenza period.

Respiratory and intensive care clinicians are therefore reminded of the current Health Protection Agency clinical 